\title{
Asymmetric Intramolecular Alkylation of Chiral Aromatic Imines via Catalytic C-H Bond Activation
}

\author{
Anja Watzke, Rebecca M. Wilson, Steven J. O'Malley, Robert G. Bergman, ${ }^{*}$ Jonathan A. Ellman* \\ Department of Chemistry and Division of Chemical Sciences, Lawrence Berkeley National Laboratory, University of California, \\ Berkeley, CA 94720 , USA \\ Fax +1(510)6427714; E-mail: rbergman@berkeley.edu; E-mail: jellman@berkeley.edu \\ Received 16 April 2007
}

Abstract: The asymmetric intramolecular alkylation of chiral aromatic aldimines, in which differentially substituted alkenes are tethered meta to the imine, was investigated. High enantioselectivities were obtained for imines prepared from aminoindane derivatives, which function as directing groups for the rhodium-catalyzed $\mathrm{C}-\mathrm{H}$ bond activation. Initial demonstration of catalytic asymmetric intramolecular alkylation also was achieved by employing a sterically hindered achiral imine substrate and catalytic amounts of a chiral amine.

Key words: $\mathrm{C}-\mathrm{H}$ bond activation, asymmetric catalysis, transimination, cyclization, dihydrobenzofuran

The intramolecular alkylation of aromatic imines via rhodium-catalyzed ortho-directed $\mathrm{C}-\mathrm{H}$ bond activation' provides an efficient route to functionalized bicyclic ring systems. ${ }^{2}$ A catalytic and highly enantioselective variant of this intramolecular alkylation has been achieved for some alkene substitution patterns using chiral phosphoramidite ligands. ${ }^{3}$ However, for other alkene substitution patterns, alternative approaches for achieving asymmetric induction may be necessary. In the context of the first total synthesis of $(+)$-lithospermic acid, ${ }^{4,5}$ the first example of employing a chiral imine as a directing group in catalytic $\mathrm{C}-\mathrm{H}$ bond activation was developed. Specifically, imine 2 , prepared by condensation of 1 with $R$-(-)-aminoindane, was cyclized to give product 3 in $76 \%$ ee after auxiliary hydrolysis (Scheme 1). Herein, improved chiral imine directing groups are reported and evaluated with substrates containing different alkene substitution patterns. ${ }^{4}$

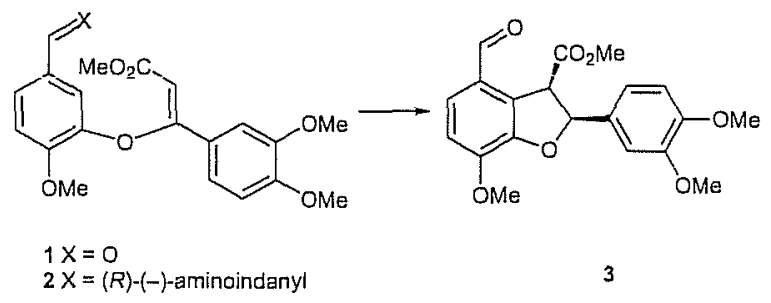

Scheme 1 Asymmetric cyclization of lithospermic acid precursor
In previously published work, we evaluated a range of structurally diverse chiral amine directing groups, with aminoindane proving to be most effective. Therefore, to further increase the stereoselectivity of the intramolecular alkylation reaction, we chose to evaluate substituted aminoindane derivatives, which were synthesized from the corresponding indanones by one-pot reductive amination with tert-butanesulfinamide (Scheme 2 and Scheme 3). ${ }^{6}$.

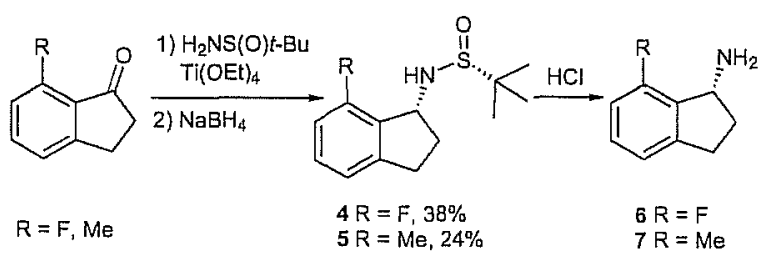

Scheme 2 Asymmetric synthesis of chiral aminoindane derivatives 6 and 7

Condensation of tert-butanesulfinamide with 7-fluoroindanone ${ }^{7}$ and 7-methylindanone and subsequent reduction of the $N$-tert-butanesulfinyl imines with $\mathrm{NaBH}_{4}$ provided sulfinamides 4 and 5 with $d r>99: 1$. The modest yields observed in these reactions are presumably the result of competing enamine formation in the imine condensation step (Scheme 2). Subsequent removal of the tert-butanesulfinyl group under acidic conditions provided the enantiomerically pure amines 6 and 7 .
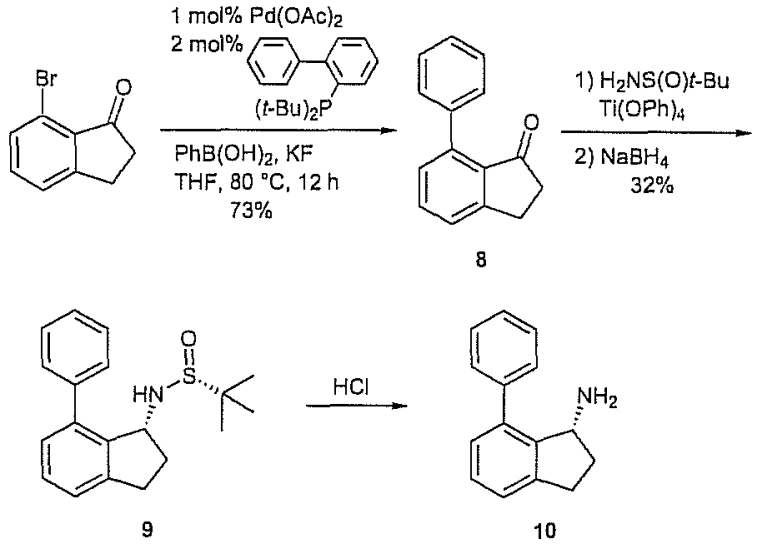

Scheme 3 Asymmetric synthesis of chiral 7-phenylaminoindane 10 
For the introduction of a phenyl group in position 7 of the aminoindane, 7-bromo-aminoindanone ${ }^{7}$ was subjected to Suzuki cross coupling under conditions described by Buchwald ${ }^{8}$ to provide 8 in high yield (Scheme 3). Attempted condensation of ketone 8 with tert-butanesulfinamide using $\mathrm{Ti}(\mathrm{OEt})_{4}$ resulted in Meerwein-PondorfVerley (MPV) reduction to give the undesired corresponding alcohol byproduct. Therefore, $\mathrm{Ti}(\mathrm{OPh})_{4},{ }^{9}$ which is not capable of participating in MPV reduction, was used instead of Ti(OEt $)_{4}$ and provided sulfinamide 9 with high selectivity albeit in modest yield. Removal of the tertbutanesulfinyl group under acidic conditions gave enantiomerically pure amine 10.

Each 7-substituted 2-aminoindane was condensed with aldehyde 1 to obtain the corresponding chiral imines 2 and 11-13. Using optimized conditions with ferrocenyl-PCy $\left(\mathrm{FCPCy}_{2}\right)$ as the ligand, the rhodium-catalyzed intramolecular alkylation led, after hydrolysis, to dihydrobenzofuran 3 with good yields and improved enantioselectivity (Table 1). The enantioselectivity provided by the 7 -fluoroaminoindane chiral auxiliary at $90 \%$ ee was particularly impressive (entry 3 ).

We next focused our efforts on the asymmetric alkylation of substrates with different alkene substitution patterns to explore the scope of this method. Cyclization of substrates 14 and 15 with a phenyl group in place of the 3,4dimethoxyphenyl group proceeded in good yields but with appreciable reduction in enantioselectivity (Table 2).

Imines 17-20 with 1,1- and 1,2-disubstituted double bonds, respectively, were also evaluated (Table 3 ). Conversion of the corresponding aldehyde into chiral imines 17 and 18 followed by cyclization provided dihydrofuran 21 in quantitative yield after auxiliary hydrolysis. Once

Table 1 Asymmetric Cyclization of Chiral Imines 2, 11-13

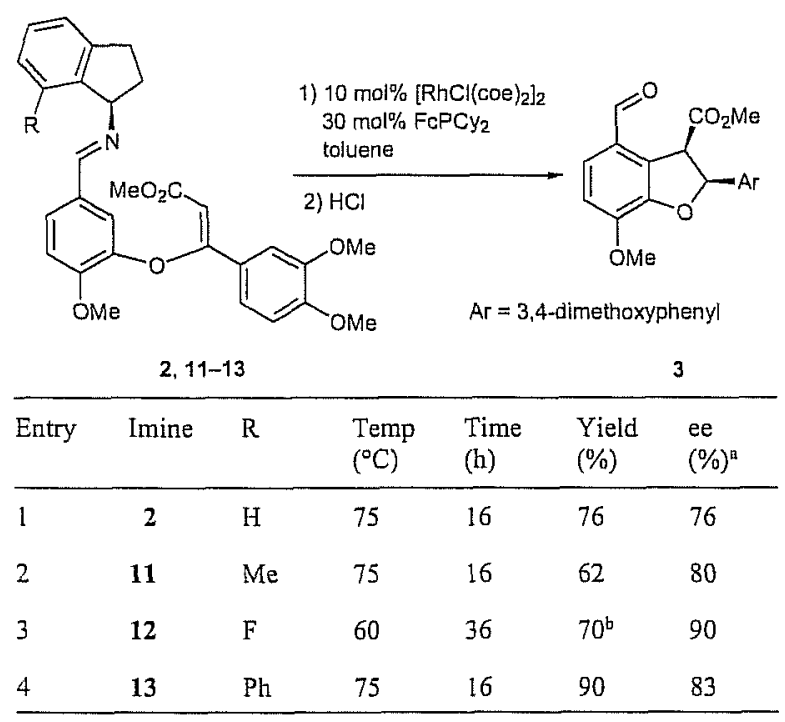

${ }^{a}$ Enantiomeric excess determined by chiral HPLC after hydrolysis of imine with $1 \mathrm{M} \mathrm{HCl}(\mathrm{aq})$.

${ }^{\mathrm{b}}$ Includes trace amounts of trans diastereomer.
Table 2 Asymmetric Cyclization of Chiral Imines 14 and 15

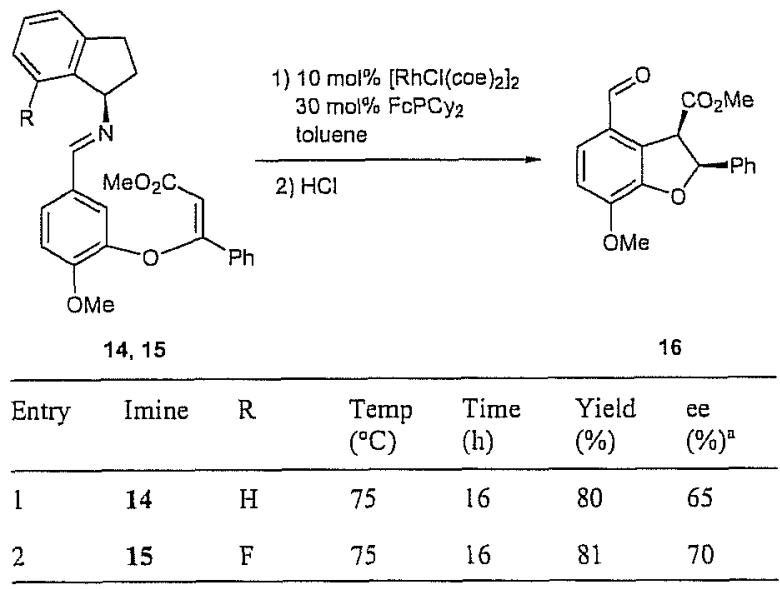

${ }^{a}$ Enantiomeric excess determined by chiral HPLC after hydrolysis of imine with $1 \mathrm{M} \mathrm{HCl}$ (aq).

Table 3 Asymmetric Cyclization of Chiral Imines 17-20

\begin{tabular}{|c|c|c|c|c|c|c|c|}
\hline \multirow{3}{*}{\multicolumn{2}{|c|}{$17-20$}} & & \multicolumn{3}{|c|}{$\begin{array}{l}\text { 1) } 10 \text { mal\% }\left[\mathrm{RhCl}(\mathrm{Goe})_{2}\right]_{2} \\
30 \text { mol\% } \mathrm{FcPCy}{ }_{2} \\
\text { toluene }\end{array}$} & & \\
\hline & & & \multirow{2}{*}{\multicolumn{3}{|c|}{ 2) $\mathrm{HCl}$}} & & \\
\hline & & & & & & & \\
\hline Entry & Imine & $\mathrm{R}^{\mathrm{l}}$ & $\mathrm{R}^{2}$ & $\mathrm{R}^{3}$ & $\begin{array}{l}\text { Yield } \\
(\%)^{\mathrm{a}}\end{array}$ & $\begin{array}{l}\text { ee } \\
(\%)^{b}\end{array}$ & Product \\
\hline 1 & 17 & $\mathrm{H}$ & $\mathrm{Me}$ & $\mathrm{H}$ & $>99$ & 58 & 21 \\
\hline 2 & 18 & $\mathrm{~F}$ & $\mathrm{Me}$ & $\mathrm{H}$ & $>99$ & 68 & 21 \\
\hline 3 & 19 & $\mathrm{H}$ & $\mathrm{H}$ & $\mathrm{Me}$ & 34 & 55 & 22 \\
\hline 4 & 20 & $\mathrm{~F}$ & $\mathrm{H}$ & $\mathrm{Me}$ & 50 & 58 & 22 \\
\hline
\end{tabular}

a Yields based on 'H NMR integration relative to 2,6-dimethoxytoluene as an internal standard.

${ }^{b}$ Enantiomeric excess determined by chiral HPLC after hydrolysis of imine with $1 \mathrm{M} \mathrm{HCl}(\mathrm{aq})$.

again, 7-fluoro-aminoindane provided the highest enantiomeric excess (entry 2). In contrast, imines 19 and 20, prepared from the 1,2-disubstituted aldehyde, cyclized significantly less efficiently, with product 22 being obtained in lower yield and with lower enantioselectivity (entries 3 and 4).

Additionally, asymmetric cyclization of an achiral imine through imine exchange ${ }^{10}$ using catalytic amounts of chiral amine was investigated. Effective imine exchange was first demonstrated by cyclization of achiral $N$-tert-butyl aldimine 23 upon addition of one equivalent of chiral aminoindane (Table 4 , entry 1). The enantiomeric purity of the product aldehyde 21 establishes that transimination 
Table 4 Catalytic Asymmetric Cyclization of Achiral Imine 24 through Imine Exchange

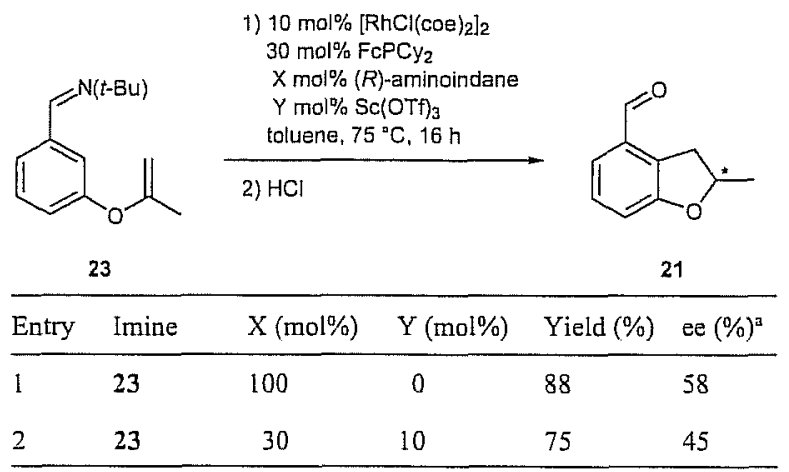

a Enantiomeric excess was determined by chiral HPLC after hydrolysis of imine with $1 \mathrm{M} \mathrm{HCl}(\mathrm{aq})$

occurs considerably faster than cyclization of the sterically hindered achiral imine, which would lead to racemic product. Catalytic asymmetric cyclization of 23 was next demonstrated by addition of $30 \mathrm{~mol} \%$ of chiral aminoindane along with $10 \mathrm{~mol} \% \mathrm{Sc}(\mathrm{OTf})_{3}$, which was required to accelerate the imine exchange process. Only a minor drop in the yield and enantiomeric excess of product 22 was observed (entry 2).

In conclusion, asymmetric intramolecular alkylation of chiral aromatic aldimines was evaluated for differentially substituted alkene substrates. The highest enantioselectivities were obtained using the 7-fluoro-aminoindane as the chiral auxiliary with cyclization providing product in $90 \%$ ee for the most favorable substrate. Additionally, initial demonstration of catalytic asymmetric intranolecular alkylation was accomplished by employing a sterically hindered achiral imine substrate and catalytic amounts of a chiral amine.

Unless otherwise noted, materials were obtained from commercial suppliers and used without further purification. THF was distilled under $\mathrm{N}_{2}$ from sodium benzophenone ketyl, pyridine from $\mathrm{CaH}_{2}$, and $\mathrm{MeOH}$ from $\mathrm{Mg}(\mathrm{MeO})_{2}$ immediately prior to use. Degassed toluene was purified by passage through an activated alumina column. All organic reactions were performed under an atmosphere of $\mathrm{N}_{2}$ in flame-dried or oven-dried glassware unless otherwise stated. All $\mathrm{C}-\mathrm{H}$ activation experiments were prepared in an $\mathrm{N}_{2}$-filled Vac inert atmosphere box. The following compounds were prepared according to referenced literature procedures: $\left[\mathrm{RhCl}(\mathrm{coe})_{2}\right]_{2}{ }^{11}$ and $\mathrm{FcPCy}_{2}{ }^{12}$ Thin-layer chromatography was performed on Merck 60 F254 $250 \mu \mathrm{m}$ silica gel plates. Visualization of the developed chromatogram was performed by fluorescence quenching, $\mathrm{KMnO}_{4}$ stain, or $p$-anisaldehyde stain. Flash chromatography was carried out according to the general procedure of Still ${ }^{13}$ using Merck 60 230-240 mesh silica gel or basic $\mathrm{Al}_{2} \mathrm{O}_{3}$ (activity grade I) as noted. Organic extracts were dried over $\mathrm{MgSO}_{4}$ or $\mathrm{Na}_{2} \mathrm{SO}_{4}$ and were concentrated using a Büchi rotary evaporator under high vacuum pressure. Melting points were determined on a Mel-Temp apparatus and are uncorrected. Unless otherwise noted, ${ }^{1} \mathrm{H}$ NMR and ${ }^{13} \mathrm{C}$ NMR spectra were obtained in $\mathrm{CDCl}_{3}$. NMR chemical shifts are reported in $\mathrm{ppm}$ and referenced to residual protonated solvent. Mass spectra were performed by the University of California, Berkeley MicroMass Facility. Chiral HPLC analyses were performed on an Agilent
1100 system with a Chiral PAK AS column $(250 \mathrm{~mm} 4.6 \mathrm{~mm})$ with a flow rate of $1 \mathrm{~mL} / \mathrm{min}$ and with $i-\mathrm{PrOH}$-hexanes as the mobile phase. A Perkin-Elmer 241 polarimeter with a sodium lamp was used to determine specific rotations and concentrations are reported in $\mathrm{g} / \mathrm{dL}$.

\section{Imine Formation}

To a $25 \mathrm{~mL}$ round-bottom flask equipped with a magnetic stir bar and septum was added $1.00 \mathrm{mmol}$ of aldehyde, benzene $(10 \mathrm{~mL})$, MS $4 \AA(1.0 \mathrm{~g})$, and 1.1 equiv of amine $(1.10 \mathrm{mmol})$. The reaction flask was then equipped with a reflux condenser, and the mixture was heated to reflux for $12 \mathrm{~h}$. The reaction mixture was cooled to r.t., diluted with benzene, dried over $\mathrm{Na}_{2} \mathrm{SO}_{4}$, filtered, and concentrated.

Cyclization of Aromatic Imines

In a dry box, a solution of $\left[\mathrm{RhCl}(\mathrm{Coe})_{2}\right]_{2}(3.59 \mathrm{mg}, 5 \mu \mathrm{mol}, 0.1$ equiv) and $\mathrm{FcPCy}_{2}\left(5.73 \mathrm{mg}, 15.0 \mu \mathrm{mol}, 0.3\right.$ equiv) in toluene- $d_{\mathrm{B}}$ $(0.25 \mathrm{~mL})$ was added to a solution of imine $(50.0 \mu \mathrm{mol}, 1.0$ equiv) in toluene $(0.25 \mathrm{~mL})$ and then transferred to an NMR tube. The tube was sealed, and the solution was stirred at $75^{\circ} \mathrm{C}$ for $16 \mathrm{~h}$. The reaction solution was cooled to r.t., transferred to a round-bottom flask with EtOAc rinses, and concentrated under reduced pressure. The residue was washed with $1 \mathrm{~N} \mathrm{HCl}(\mathrm{aq})$ and stirred for $20 \mathrm{~min}$. The mixture was diluted with EtOAc, the layers were separated, and the aqueous layer was extracted with EtOAc. The combined organic layers were dried, filtered, and concentrated under reduced pressure. The crude material was chromatographed on $\mathrm{SiO}_{2}$.

\section{Deprotection of tert-Butanesulfinamide Derivatives}

To the tert-butanesulfinamide derivatives was added $1: 1(\mathrm{v} / \mathrm{v})$ $\mathrm{MeOH}$ and $\mathrm{HCl}-$ dioxane solution ( $4 \mathrm{M}, 3$ equiv). The mixture was stirred at r.t. for $30 \mathrm{~min}$ and then concentrated to near dryness. The amine hydrochloride was precipitated in a mixture of $\mathrm{MeOH}$ and tert-butyl methyl ether. The precipitate was then filtered off and washed with tert-butyl methyl ether. The amine hydrochloride was dissolved in $1 \mathrm{~N} \mathrm{NaHCO}_{3}$ solution, which was subsequently extracted three times with EtOAc. The combined organic layers were dried over $\mathrm{MgSO}_{4}$, filtrated, and concentrated to provide each amine in quantitative yields.

3-(3,4-Dimethoxyphenyl)-3-[5-(indan-1-yliminomethyl)-2methoxyphenoxy]acrylic Acid Methyl Ester (2)

This compound was prepared according to the general procedure for imine formation with 1 equiv of aldehyde $(18.6 \mathrm{mg}, 0.0500 \mathrm{mmol})$ and 1.5 equiv of amine $(7.32 \mathrm{mg}, 0.0550 \mathrm{mmol})$. 'H NMR (400 $\left.\mathrm{MHz}, \mathrm{CDCl}_{3}\right): \delta=8.18(\mathrm{~s}, 1 \mathrm{H}, \mathrm{CH}=\mathrm{NCH}), 7.38(\mathrm{~d}, 1 \mathrm{H}, J=8.4 \mathrm{~Hz}$, ArH), 7.31-7.11 (m, 6 H, ArH x 6), 7.00 (d, $1 \mathrm{H}, J=7.3 \mathrm{~Hz}, \mathrm{ArH}$ ), 6.90 (d, $1 \mathrm{H}, J=8.4 \mathrm{~Hz}, \mathrm{ArH}), 6.73(\mathrm{~d}, 1 \mathrm{H}, J=8.5 \mathrm{~Hz}, \mathrm{ArH}), 5.97$ (s, $\left.1 \mathrm{H}, \mathrm{CHCO}_{2} \mathrm{CH}_{3}\right), 4.78\left(\mathrm{t}, 1 \mathrm{H}, J=7.2 \mathrm{~Hz}, \mathrm{CH}_{2} \mathrm{CHN}=\mathrm{CH}\right.$ ), 3.93 $\left(\mathrm{s}, 3 \mathrm{H}, \mathrm{OCH}_{3}\right), 3.79\left(\mathrm{~s}, 3 \mathrm{H}, \mathrm{OCH}_{3}\right), 3.76\left(\mathrm{~s}, 3 \mathrm{H}, \mathrm{OCH}_{3}\right), 3.64(\mathrm{~s}, 3$ $\left.\mathrm{H}, \mathrm{CO}_{2} \mathrm{CH}_{3}\right), 3.07-3.01\left(\mathrm{~m}, 1 \mathrm{H}\right.$, one of $\left.\mathrm{ArCH}_{2} \mathrm{CH}_{2}\right), 2.92-2.84(\mathrm{~m}$, $1 \mathrm{H}$, one of $\left.\mathrm{ArCH}_{2} \mathrm{CH}_{2}\right), 2.39-2.35\left(\mathrm{~m}, 1 \mathrm{H}\right.$, one of $\left.\mathrm{ArCH}_{2} \mathrm{CH}_{2}\right)$, 2.19-2.09 (m, $1 \mathrm{H}$, one of $\left.\mathrm{ArCH}_{2} \mathrm{CH}_{2}\right)$. ${ }^{13} \mathrm{C}$ NMR (100 MHz, $\left.\mathrm{CDCl}_{3}\right): \delta=164.6,162.6,159.0,151.3,150.8,148.6,146.0,144.1$ $143.4,129.2,128.0,127.2,126.0,124.4,124.0,124.0,120.5,116.1$ $111.6,110.6,109.5,103.6,74.5,55.8,55.5,55.4,50.9,33.9,30.7$ $[a]_{D}^{25} 7.7\left(c 1.12, \mathrm{CH}_{2} \mathrm{Cl}_{2}\right)$. HRMS-FAB: m/z calcd for $\mathrm{C}_{29} \mathrm{H}_{30} \mathrm{NO}_{6}$ $[\mathrm{M}+\mathrm{H}]^{+}:$: 488.2073; found: 488.2079 .

2-Methylpropane-2-sulfinic Acid (7-Fluoroindan-1-yl)amide (4)

To a $25 \mathrm{~mL}$ round-bottom flask equipped with a magnetic stir bar and septum were added 7-fluoroindan-1-one (190 $\mathrm{mg}, 1.23 \mathrm{mmol}$ ), 2 equiv of (R)-2-methylpropane-2-sulfinic acid amide $(297 \mathrm{mg}$, $2.45 \mathrm{mmol}$ ), and $2.5 \mathrm{~mL}$ of THF. Under argon atmosphere, 4 equiv of $\mathrm{Ti}(\mathrm{OEt})_{4}(1.12 \mathrm{~g}, 4.90 \mathrm{mmol})$ was added to the reaction mixture. 
The reaction flask was then equipped with a reflux condenser and the mixture was heated to reflux for $12 \mathrm{~h}$. The reaction mixture was cooled to $-78^{\circ} \mathrm{C}$ and 4 equiv of $\mathrm{NaBH}_{4}(185 \mathrm{mg}, 4.90 \mathrm{mmol})$ was added. The reaction mixture was stirred for $30 \mathrm{~min}$ at $-78^{\circ} \mathrm{C}$ and allowed to warm to r.t. The reaction mixture was quenched with $\mathrm{NaCl}$ solution, and the precipitate was filtered off and washed with EtOAc. After phase separation, the aqueous phase was extracted with EtOAc. The combined organic phases were washed with brine, dried over $\mathrm{Na}_{2} \mathrm{SO}_{4}$, filtered, and concentrated. The crude material was chromatographed on $\mathrm{SiO}_{2}(10-50 \%$ EtOAc-hexanes) to afford compound 4 as a yellow oil (120 mg, $0.470 \mathrm{mmol}, 38 \%$ ). 'H NMR (400 MHz, $\left.\mathrm{CDCl}_{3}\right): \delta=7.19-7.14(\mathrm{~m}, 1 \mathrm{H}, \mathrm{ArH}), 6.97(\mathrm{~d}, 1 \mathrm{H}$, $J=7.2 \mathrm{~Hz}, \mathrm{ArH}), 6.81(\mathrm{t}, 1 \mathrm{H}, J=8.8 \mathrm{~Hz}, \mathrm{ArH}), 5.08-5.05(\mathrm{~m}, 1 \mathrm{H}$, $\left.\mathrm{HNCHCH} \mathrm{CH}_{2}\right), 3.76$ [br s, $1 \mathrm{H}$, NHS(O)t-Bu], 3.11-3.03 (m, $1 \mathrm{H}$, one of $\mathrm{ArCH}_{2} \mathrm{CH}_{2}$ ), 2.86-2.79 (m, I H, one of $\mathrm{ArCH}_{2} \mathrm{CH}_{2}$ ), 2.44 $2.35\left(\mathrm{~m}, 1 \mathrm{H}\right.$, one of $\left.\mathrm{ArCH}_{2} \mathrm{CH} \mathrm{H}_{7}\right), 2.19-2.11(\mathrm{~m}, 1 \mathrm{H}$, one of $\left.\mathrm{ArCH}_{2} \mathrm{CH}_{2}\right), 1.12\left[\mathrm{~s}, 9 \mathrm{H}, \mathrm{C}\left(\mathrm{CH}_{3}\right)_{3}\right],{ }^{13} \mathrm{C} \mathrm{NMR}\left(100 \mathrm{MHz}, \mathrm{CDCl}_{3}\right)$ : $\delta=160.5,158.0,147.5,147.4,130.1,130.1,128.9,128.8,120.5$, $120.4,113.2,113.0,56.7,56.7,55.3,33.3,30.6,30.6,22.2$ (multiple $\mathrm{C}^{\prime} \mathrm{s}$ coupled to $\left.\mathrm{F}\right)$. $[\alpha]_{\mathrm{D}}{ }^{25}-6.1\left(\mathrm{c} 2.8, \mathrm{CH}_{2} \mathrm{Cl}_{2}\right)$. HRMS-FAB: $\mathrm{m} / \mathrm{z}$ caled for $\mathrm{C}_{13} \mathrm{H}_{19}$ FNOS [M+ H] $]^{+}: 256.1171$; found: 256.1171 .

Propane-2-sulfinic Acid (7-Methylindan-1-yl)amide (5)

To a $25 \mathrm{~mL}$ round-bottom flask equipped with a magnetic stir bar and septum were added 7-methylindan-1-one (179 mg, $1.23 \mathrm{mmol})$, 2 equiv of (R)-2-methylpropane-2-sulfinic acid amide $(297 \mathrm{mg}$, $2.45 \mathrm{mmol}$ ), and $2.5 \mathrm{~mL}$ of THF. Under argon atmosphere, 4 equiv of $\mathrm{Ti}(\mathrm{OEt})_{4}(1.12 \mathrm{~g}, 4.90 \mathrm{mmol})$ was added to the reaction mixture. The reaction flask was then equipped with a reflux condenser and the mixture was heated to reflux for $12 \mathrm{~h}$. The reaction mixture was cooled to $-78{ }^{\circ} \mathrm{C}$ and 4 equiv of $\mathrm{NaBH}_{4}(185.4 \mathrm{mg}, 4.90 \mathrm{mmol})$ was added. The reaction mixture was stirred for $30 \mathrm{~min}$ at $-78^{\circ} \mathrm{C}$ and allowed to warm to r.t. The reaction mixture was quenched with $\mathrm{NaCl}$ solution, and the precipitate was filtered off and washed with EtOAc. After phase separation, the aqueous phase was extracted with EtOAc. The combined organic phases were washed with brine, dried over $\mathrm{Na}_{2} \mathrm{SO}_{4}$, filtered, and concentrated. The crude material was chromatographed on $\mathrm{SiO}_{2}(10-50 \%$ EtOAc-hexanes) to afford compound 5 as a white solid ( $73.9 \mathrm{mg}, 0.294 \mathrm{mmol}, 24 \%) ; \mathrm{mp} 67-$ $68^{\circ} \mathrm{C}$. 'H NMR ( $\left.400 \mathrm{MHz}, \mathrm{CDCl}_{3}\right): \delta=7.17$ (t, $1 \mathrm{H}, J=7.2 \mathrm{~Hz}$ ArH), 7.08 (d, $1 \mathrm{H}, J=7.6 \mathrm{~Hz}, \mathrm{ArH}), 6.99$ (d, $1 \mathrm{H}, J=7.2 \mathrm{~Hz}, \mathrm{ArH})$, 4.94 (br s, I H, $\mathrm{FNCHCH} \mathrm{CH}_{2}$ ), 3.18-3.09 [m, $2 \mathrm{H}$, NHS(O) $t$-Bu, one of $\mathrm{ArCH}_{2} \mathrm{CH}_{2}$ ], 2.83-2.77 (m, $1 \mathrm{H}$, one of $\left.\mathrm{ArCH}_{2} \mathrm{CH}_{2}\right), 2.37$ (s, $\left.3 \mathrm{H}, \mathrm{ArCH}_{3}\right), 2.21-2.16\left(\mathrm{~m}, 2 \mathrm{H}, \mathrm{ArCH}_{2} \mathrm{CH}_{2}\right), 1.15[\mathrm{~s}, 9 \mathrm{H}$, $\left.\mathrm{C}\left(\mathrm{CH}_{3}\right)_{3}\right] \cdot{ }^{13} \mathrm{C}$ NMR $\left(100 \mathrm{MHz}, \mathrm{CDCl}_{3}\right): \delta=145.0,141.3,134.5$ $128.8,128.0,122.2,58.2,55.3,33.6,30.3,22.4,18.4 .[\alpha]_{D}{ }^{25}-9.2(c$ 1.5, $\mathrm{CH}_{2} \mathrm{Cl}_{2}$ ). HRMS-FAB: $\mathrm{m} / \mathrm{z}$ calcd for $\mathrm{C}_{14} \mathrm{H}_{22} \mathrm{NOS}[\mathrm{M}+\mathrm{H}]^{+}$: 252.1422; found: 252.1422 .

Amines 6 and 7 were prepared according to the general procedure for the deprotection of tert-butanesulfinamide derivatives.

\section{7-Fluoroindan-1-ylamine (6)}

'H NMR (400 MHz, CDCl $)$ ): $\delta=7.18-7.11(\mathrm{~m}, 1 \mathrm{H}, \mathrm{ArH}), 6.96$ (d, $1 \mathrm{H}, J=10.0 \mathrm{~Hz}, \mathrm{ArH}), 6.82(\mathrm{t}, 1 \mathrm{H}, J=12.0 \mathrm{~Hz}, \mathrm{ArH}), 4.63(\mathrm{t}, 1$ $\left.\mathrm{H}, J=8.4 \mathrm{~Hz}, \mathrm{H}_{2} \mathrm{NCHCH}\right), 3.08-3.01\left(\mathrm{~m}, 1 \mathrm{H}\right.$, one of $\left.\mathrm{ArCH}_{2} \mathrm{CH}_{2}\right)$, 2.86-2.76 (m, 1 H, one of $\left.\mathrm{ArCH}_{2} \mathrm{CH}_{2}\right), 2.25-2.41(\mathrm{~m}, 1 \mathrm{H}$, one of $\left.\mathrm{ArCH}_{2} \mathrm{CH}_{2}\right), 1.95-1.75\left(\mathrm{~m}, 3 \mathrm{H}\right.$, one of $\left.\mathrm{ArCH}_{2} \mathrm{CH}_{2}, \mathrm{NH}_{2}\right) .{ }^{13} \mathrm{CNMR}$ $\left(100 \mathrm{MHz}, \mathrm{CDCl}_{3}\right): \delta=161.2,158.7,146.7,146.6,133.3,133.1$, $129.3,129.2,120.5,113.2,113.0,55.0,35.5,30.6$ (multiple Cs coupled to $F) .[\alpha]_{D}^{25}-0.4\left(c 3.5, \mathrm{CH}_{2} \mathrm{Cl}_{2}\right)$

\section{7-Methylindan-1-ylamine (7)}

${ }^{1} \mathrm{H} \mathrm{NMR}\left(400 \mathrm{MHz}, \mathrm{CDCl}_{3}\right): \delta=7.17-7.05(\mathrm{~m}, 2 \mathrm{H}, \mathrm{ArH} \times 2), 6.99$ (d, $1 \mathrm{H}, J=9.2 \mathrm{~Hz}, \mathrm{ArH}), 4.50-4.46$ (dd, $1 \mathrm{H}, J=9.6,3.6 \mathrm{~Hz}$, $\left.\mathrm{H}_{2} \mathrm{NCHCH}_{2}\right), 3.17-3.06\left(\mathrm{~m}, 1 \mathrm{H}\right.$, one of $\left.\mathrm{ArCH}_{2} \mathrm{CH}_{2}\right), 2.842 .75(\mathrm{~m}$, $1 \mathrm{H}$, one of $\left.\mathrm{ArCH} \mathrm{CH}_{2}\right), 2.40\left(\mathrm{~s}, 3 \mathrm{H}, \mathrm{ArCH}_{3}\right), 2.40-2.29(\mathrm{~m}, 1 \mathrm{H}$, one of $\left.\mathrm{ArCH}_{2} \mathrm{CH}_{2}\right), 1.96-1.88\left(\mathrm{~m}, 1 \mathrm{H}\right.$, one of $\left.\mathrm{ArCH}_{2} \mathrm{CH}_{2}\right), 1.55$ (br s, $\left.2 \mathrm{H}, \mathrm{NH}_{2}\right),{ }^{13} \mathrm{C} \mathrm{NMR}\left(100 \mathrm{MHz}, \mathrm{CDCl}_{3}\right): \delta=145.3,143.2,133.9$, $127.9,127.7,122.3,55.9,35.7,30.2,18.4$. $[0 .]_{D}{ }^{25}-2.08$ (c 0.5 , $\mathrm{CH}_{2} \mathrm{Cl}_{2}$ ).

\section{7-Phenylindan-1-0ne (8)}

In a dry box, 7-bromoindan-1-one (105 mg, $0.500 \mathrm{nmol}), 3$ equiv of KF (87.2 $\mathrm{mg}, 1.50 \mathrm{mmol}), 1.5$ equiv of phenylboronic acid $(91.5$ $\mathrm{mg}, 0.750 \mathrm{mmol}), 1 \mathrm{~mol} \%$ of $\mathrm{Pd}(\mathrm{OAc})_{2}(1.1 \mathrm{mg}, 0.0050 \mathrm{mmol})$, $2 \mathrm{~mol} \%$ of biph $(t-\mathrm{Bu})_{2}(3.0 \mathrm{mg}, 0.010 \mathrm{mmol})$, and $0.5 \mathrm{~mL}$ of THF were added to a Schlenk flask. The flask was sealed, and the solution was stirred at $80^{\circ} \mathrm{C}$ for $14 \mathrm{~h}$. The reaction solution was cooled to r.t., diluted with EtOAc, and washed with brine and $\mathrm{H}_{2} \mathrm{O}$. The aqueous layer was extracted with EtOAc, and the combined organic layers were dried, filtered, and concentrated under reduced pressure. The crude material was chromatographed on $\mathrm{SiO}_{2}(0-10 \%$ EtOAc-hexanes) to afford compound 8 as a white solid $(76.0 \mathrm{mg}$, $0.365 \mathrm{mmol}, 73 \%)$; mp 91-92 ${ }^{\circ} \mathrm{C}$. ${ }^{1} \mathrm{H}$ NMR $\left(400 \mathrm{MHz}, \mathrm{CDCl}_{3}\right): \delta=$ $7.60(\mathrm{t}, 1 \mathrm{H}, J=7.6 \mathrm{~Hz}, \mathrm{ArH}), 7.52-7.4 \mathrm{l}(\mathrm{m}, 6 \mathrm{H}, \mathrm{ArH} \times 6), 7.28(\mathrm{~d}$ $1 \mathrm{H}, J=7.6 \mathrm{~Hz}, \mathrm{ArH}), 3.16-3.13\left(\mathrm{~m}, 2 \mathrm{H}, \mathrm{ArCH}_{2} \mathrm{CH}_{2}\right), 2.71-2.68$ (m, $\left.2 \mathrm{H}, \mathrm{ArCH}_{2} \mathrm{CH}_{2}\right),{ }^{13} \mathrm{C}$ NMR $\left(100 \mathrm{MHz}, \mathrm{CDCl}_{3}\right): \delta=205.2$, $156.1,141.1,137.8,133.6,132.7,129.1,129.1,127.5,127.4,125.5$, 36.6, 25.0. HRMS-FAB: $m / 2$ calcd for $\mathrm{C}_{15} \mathrm{H}_{13} \mathrm{O}[\mathrm{M}+\mathrm{H}]^{+}$: 209.0966; found: 209.0966 .

\section{2-Methylpropane-2-sulfinic Acid (7-phenylindan-1-yl)amide} (9)

To a $25 \mathrm{~mL}$ round-bottom flask equipped with a magnetic stir bar and septum were added 7-phenylindan-1-one $(200 \mathrm{mg}, 0.960$ mmol), 2 equiv of $(R)$-2-methylpropane-2-sulfinic acid amide (233 $\mathrm{mg}, 1.92 \mathrm{mmol}$ ), and $2.5 \mathrm{~mL}$ of THF. Under an argon atmosphere, 4 equiv of $\mathrm{Ti}(\mathrm{OPh})_{4}(1.61 \mathrm{~g}, 3.84 \mathrm{mmol})$ was added to the reaction mixture. The reaction flask was then equipped with a reflux condenser, and the mixture was heated to reflux for $12 \mathrm{l}$. The reaction mixture was then cooled to $-78{ }^{\circ} \mathrm{C}$, and 4 equiv of $\mathrm{NaBH}_{4}(145 \mathrm{mg}$, $3.84 \mathrm{mmol}$ ) were added. The reaction mixture was stirred for $30 \mathrm{~min}$ at $-78^{\circ} \mathrm{C}$ and allowed to warm to r.t. The reaction mixture was quenched with $\mathrm{NaCl}$ solution, and the precipitate was filtered off and washed with EtOAc. After phase separation, the aqueous phase was extracted with EtOAc. The combined organic phases were washed with brine, dried over $\mathrm{Na}_{2} \mathrm{SO}_{4}$, filtered, and concentrated. The crude material was chromatographed on $\mathrm{SiO}_{2}(10-40 \%$ EtOAc-hexanes) to afford compound 9 as a white solid $(95.0 \mathrm{mg}$, $0.303 \mathrm{mmol}, 32 \%)$; mp 109-110 ${ }^{\circ} \mathrm{C}$. 'H NMR (400 MHz, CDCl $)$ : $\delta=7.51-7.45(\mathrm{~m}, 4 \mathrm{H}, \mathrm{ArH} \times 4), 7.36-7.41(\mathrm{~m}, 1 \mathrm{H}, \mathrm{ArH}), 7.33(\mathrm{~d}$, $1 \mathrm{H}, J=7.6 \mathrm{~Hz}, \mathrm{ArH}), 7.27-7.29(\mathrm{~m}, 1 \mathrm{H}, \mathrm{ArH}), 7.18(\mathrm{~d}, 1 \mathrm{H}$, $J=7.2 \mathrm{~Hz}, \mathrm{ArH}), 5.29\left(\mathrm{t}, 1 \mathrm{H}, J=6.0 \mathrm{~Hz}, \mathrm{HNCHCH}_{2}\right), 3.42[\mathrm{~s}, 1 \mathrm{H}$, NHS(O)t-Bu], 3.14-3.08 (m, $1 \mathrm{H}$, one of $\left.\mathrm{ArCH}_{2} \mathrm{CH}_{2}\right), 3.01-2.93$ (m, $1 \mathrm{H}$, one of $\mathrm{ArCH}_{2} \mathrm{CH}_{2}$ ), 2.60-2.53 (m, $1 \mathrm{H}$, one of $\mathrm{ArCH}_{2} \mathrm{CH}_{2}$ ), 2.14-2.07 (m, $1 \mathrm{H}$, one of $\left.\mathrm{ArCH}_{2} \mathrm{CH}_{2}\right), 0.89\left[\mathrm{~s}, 9 \mathrm{H}, \mathrm{C}\left(\mathrm{CH}_{3}\right)_{3}\right] \cdot{ }^{13} \mathrm{C}$ NMR $\left(100 \mathrm{MHz}, \mathrm{CDCl}_{3}\right): \delta=144.8,140.4,139.7,138.6,129.0$, $128.4,128.4,127.8,127.8,124.0,57.3,54.8,32.7,30.5,22.1 .[\alpha]_{D}{ }^{25}$ -16.8 (c 0.6, $\mathrm{CH}_{2} \mathrm{Cl}_{2}$ ). HRiviS-FAB: $m / z$ caicd for $\mathrm{C}_{19} \mathrm{H}_{24} \mathrm{NOS}$ $[\mathrm{M}]^{+}: 314.1579$; found: 314.1579 .

\section{7-Phenylindan-1-ylamine (10)}

This compound was prepared according to the general procedure for the deprotection of tert-butanesulfinamide derivatives. ${ }^{1} \mathrm{H}$ NMR $\left(400 \mathrm{MHz}, \mathrm{CDCl}_{3}\right): \delta=7.49-7.44(\mathrm{~m}, 4 \mathrm{H}, \mathrm{ArH} \times 4), 7.38-7.36(\mathrm{~m}$, $1 \mathrm{H}, \mathrm{ArH}), 7.31-7.22(\mathrm{~m}, 2 \mathrm{H}, \mathrm{ArH} \times 2), 7.07$ (d, $1 \mathrm{H}, J=10.0 \mathrm{~Hz}$, ArH), 4.74 (t, $1 \mathrm{H}, J=8.4 \mathrm{~Hz}, \mathrm{H}_{2} \mathrm{NCHCH}_{2}$ ) $3.14-3.08$ (m, $1 \mathrm{H}$, one of $\mathrm{ArCH}_{2} \mathrm{CH}_{2}$ ), 3.13-3.03 (m, I $\mathrm{H}$, one of $\mathrm{ArCH}_{2} \mathrm{CH}_{2}$ ), 2.94-2.84 (m, $1 \mathrm{H}$, one of $\left.\mathrm{ArCH} \mathrm{CH}_{2}\right), 2.54-2.43\left(\mathrm{~m}, 1 \mathrm{H}\right.$, one of $\mathrm{ArCH}_{2} \mathrm{CH}_{2}$ ), $1.81-1.70\left(\mathrm{~m}, 1 \mathrm{H}\right.$, one of $\left.\mathrm{ArCH}_{2} \mathrm{CH}_{2}\right), 1.47$ (br s, $\left.2 \mathrm{H}, \mathrm{NH}_{2}\right) \cdot{ }^{13} \mathrm{C}$ NMR $\left(100 \mathrm{MHz}, \mathrm{CDCl}_{3}\right): \delta=143.9,140.8,138.7,128.6,128.3$, $127.8,127.7,127.2,124.0,56.1,35.2,30.2$. $[\alpha]_{D}{ }^{25}-3.5$ (c 3.5, $\mathrm{CH}_{2} \mathrm{Cl}_{2}$ ). 
3-(3,4-Dimethoxyphenyl)-3-\{2-methoxy-5-[(7-methylindan-1ylimino)methyl]phenoxy\}acrylic Acid Methyl Ester (11)

This compound was prepared according to the general procedure for imine synthesis with 1 equiv of aldehyde $(18.6 \mathrm{mg}, 0.0500 \mathrm{mmol})$ and 1.5 equiv of amine ( $8.1 \mathrm{mg}, 0.0550 \mathrm{mmol})$. 'H NMR ( $400 \mathrm{MHz}$, $\left.\mathrm{CDCl}_{3}\right): \delta=8.08(\mathrm{~s}, 1 \mathrm{H}, \mathrm{CH}=\mathrm{NCH}), 7.33(\mathrm{~d}, 1 \mathrm{H}, J=8.5 \mathrm{~Hz}, \mathrm{ArH})$ 7.20-7.18 (m, $2 \mathrm{H}, \mathrm{ArH} \times 2), 7.15-7.09(\mathrm{~m}, 3 \mathrm{H}, \mathrm{ArH} \times 3), 6.94$ (d, $1 \mathrm{H}, J=7.0 \mathrm{~Hz}, \mathrm{ArH}), 6.80(\mathrm{~d}, 1 \mathrm{H}, J=8.5 \mathrm{~Hz}, \mathrm{ArH}), 6.77(\mathrm{~d}, 1 \mathrm{H}$, $J=8.5 \mathrm{~Hz}, \mathrm{ArH}), 5.96\left(\mathrm{~s}, \mathrm{I} \mathrm{H}, \mathrm{CHCO}_{2} \mathrm{CH}_{3}\right), 4.98-4.95(\mathrm{~m}, 1 \mathrm{H}$, $\left.\mathrm{CH}_{2} \mathrm{CHN}=\mathrm{CH}\right), 3.96\left(\mathrm{~s}, 3 \mathrm{H}, \mathrm{OCH}_{3}\right), 3.90\left(\mathrm{~s}, 3 \mathrm{H}, \mathrm{OCH}_{3}\right), 3.79(\mathrm{~s}$, $\left.3 \mathrm{H}, \mathrm{OCH}_{3}\right), 3.67\left(\mathrm{~s}, 3 \mathrm{H}, \mathrm{CO}_{2} \mathrm{CH}_{3}\right), 3.14-3.10(\mathrm{~m}, 1 \mathrm{H}$, one of $\left.\mathrm{ArCH}_{2} \mathrm{CH}_{2}\right), 2.89-2.86\left(\mathrm{~m}, 1 \mathrm{H}\right.$, one of $\left.\mathrm{ArCH}_{2} \mathrm{CH}_{2}\right), 2.39-2.34(\mathrm{~m}$, $1 \mathrm{H}$, one of $\left.\mathrm{ArCH}_{2} \mathrm{CH}_{2}\right), 2.10\left(\mathrm{~s}, 3 \mathrm{H}, \mathrm{ArCH}_{3}\right), 2.06-2.02(\mathrm{~m}, 1 \mathrm{H}$, one of $\left.\mathrm{ArCH}_{2} \mathrm{CH}_{2}\right) \cdot{ }^{13} \mathrm{C} \mathrm{NMR}\left(100 \mathrm{MHz}, \mathrm{CDCl}_{3}\right): \delta=165.0,162.9$, $158.2,151.4,151.0,148.8,146.2,144.6,142.0,135.4,129.6,128.3$, $127.9,127.8,126.4,124.1,122.2,120.7,116.3,111.8,110.8,109.8$, $103.9,73.7,56.1,55.9,55.7,51.2,33.9,31.0,18.8 \cdot[\alpha]_{\mathrm{D}}^{25}+4.2(c$ 2.0, $\mathrm{CH}_{2} \mathrm{Cl}_{2}$ ).

3-(3,4-Dimethoxyphenyl)-3-\{5-I(7-fluoroindan-1-ylimino)methyll-2-methoxyphenoxy\} acrylic Acid Methyl Ester (12)

In a dry box, 1 equiv of aldehyde $(20.8 \mathrm{mg}, 0.0559 \mathrm{mmol}), 1.1$ equiv of amine $(9.3 \mathrm{mg}, 0.0615 \mathrm{mmol})$, and $0.5 \mathrm{~mL}$ of benzene were combined in a vial and stirred overnight at r.t. The resulting reaction mixture was diluted with $2 \mathrm{~mL}$ of $\mathrm{Et}_{2} \mathrm{O}$, filtered through Celite, and concentrated to yield a colorless oil ( $28.0 \mathrm{mg}, 0.0554 \mathrm{mmol}, 99 \%)$. ${ }^{\prime} \mathrm{H}$ NMR (400 MHz, $\left.\mathrm{CDCl}_{3}\right): \delta=8.16(\mathrm{~s}, 1 \mathrm{H}, \mathrm{CH}=\mathrm{NCH}), 7.38-$ $7.36(\mathrm{~m}, 2 \mathrm{H}, \mathrm{ArH} \times 2), 7.27-7.10(\mathrm{~m}, 3 \mathrm{H}, \mathrm{ArH} \times 3), 7.04(\mathrm{~d}, 1 \mathrm{H}$, $J=7.2 \mathrm{~Hz}, \operatorname{ArH}), 6.90(\mathrm{~d}, 1 \mathrm{H}, J=8.4 \mathrm{~Hz}, \mathrm{ArH}), 6.82-6.76(\mathrm{~m}, 2$ $\mathrm{H}, \operatorname{ArH} \times 2), 5.96\left(\mathrm{~s}, 1 \mathrm{H}, \mathrm{CHCO}_{2} \mathrm{CH}_{3}\right), 5.05(\mathrm{~m}, 1 \mathrm{H}$, $\left.\mathrm{CH}_{2} \mathrm{C} H \mathrm{~N}=\mathrm{CH}\right), 3.95\left(\mathrm{~s}, 3 \mathrm{H}, \mathrm{OCH}_{3}\right), 3.87\left(\mathrm{~s}, 3 \mathrm{H}, \mathrm{OCH}_{3}\right), 3.79(\mathrm{~s}$, $\left.3 \mathrm{H}, \mathrm{OCH}_{3}\right), 3.67\left(\mathrm{~s}, 3 \mathrm{H}, \mathrm{CO}_{2} \mathrm{CH}_{3}\right), 3.21-3.17(\mathrm{~m}, 1 \mathrm{H}$, one of $\left.\mathrm{ArCH}_{2} \mathrm{CH}_{2}\right), 2.95-2.85$ (m, $1 \mathrm{H}$, one of $\left.\mathrm{ArCH}_{2} \mathrm{CH}_{2}\right), 2.45-2.35$ (m, $1 \mathrm{H}$, one of $\left.\mathrm{ArCH}_{2} \mathrm{CH}_{2}\right), 2.25-2.15\left(\mathrm{~m}, 1 \mathrm{H}\right.$, one of $\left.\mathrm{ArCH}_{2} \mathrm{CH}_{2}\right){ }^{33} \mathrm{C}$ NMR $\left(100 \mathrm{MHz}, \mathrm{CDCl}_{3}\right): \delta=165.0,163.0,161.6,159.1,151.4$, $151.0,148.7,148.1,148.0,146.1,130.1,130.0,129.7,129.7,129.5$, $128.3,126.3,124.0,120.7,120.4,120.4,116.7,113.2,113.0,111.8$, $110.8,109.7,103.7,71.6,71.6,56.1,56.0,55.9,55.8,55.7,51.2$, 34.0, 31.4 (multiple C's coupled to F).

3-(3,4-Dimethoxyphenyl)-3-\{2-methoxy-5-I(7-phenylindan-1ylimino)methylphenoxy\}acrylic Acid Methyl Ester (13) This compound was prepared according the general procedure for imine synthesis with 1 equiv of aldehyde $(18.6 \mathrm{mg}, 0.0500 \mathrm{mmol})$ and 1.5 equiv of amine $(11.5 \mathrm{mg}, 0.0550 \mathrm{mmol}$ ). ' $\mathrm{H}$ NMR (400 $\left.\mathrm{MHz}, \mathrm{CDCl}_{3}\right) ; \delta=7.37-7.05(\mathrm{~m}, 13 \mathrm{H}, \mathrm{ArH} \times 12, \mathrm{CH}=\mathrm{NCH}), 6.83$ $(\mathrm{m}, 2 \mathrm{H}, \mathrm{ArH} \times 2), 5.96\left(\mathrm{~s}, 1 \mathrm{H}, \mathrm{CHCO} \mathrm{CH}_{3}\right), 4.93(\mathrm{~m}, 1 \mathrm{H}$, $\mathrm{CH}_{2} \mathrm{CHN}=\mathrm{CH}$ ), $3.94\left(\mathrm{~s}, 3 \mathrm{H}, \mathrm{OCH}_{3}\right), 3.87\left(\mathrm{~s}, 3 \mathrm{H}, \mathrm{OCH}_{3}\right), 3.79$ (s, $\left.3 \mathrm{H}, \mathrm{OCH}_{3}\right), 3.69\left(\mathrm{~s}, 3 \mathrm{H}, \mathrm{CO}_{2} \mathrm{CH}_{3}\right), 3.20-3.18(\mathrm{~m}, 1 \mathrm{H}$, one of $\left.\mathrm{ArCH} \mathrm{CH}_{2}\right), 3.00-2.90\left(\mathrm{~m}, 1 \mathrm{H}\right.$, one of $\left.\mathrm{ArCH}_{2} \mathrm{CH}_{2}\right), 2.41-2.32(\mathrm{~m}$, $1 \mathrm{H}$, one of $\left.\mathrm{ArCH}_{2} \mathrm{CH}_{2}\right), 2.15-2.00\left(\mathrm{~m}, 1 \mathrm{H}\right.$, one of $\left.\mathrm{ArCH}_{2} \mathrm{CH}_{2}\right)$. $[\mathrm{c}]_{\mathrm{D}}{ }^{25}+7.7\left(\mathrm{c} 1.5, \mathrm{CH}_{2} \mathrm{Cl}_{2}\right)$.

2-(3,4-Dimethoxyphenyl)-4-formyl-7-methoxy-2,3-dihydrobenzofuran-3-carboxylic Acid Methyl Ester (3)

The $\mathrm{C}-\mathrm{H}$ activation reactions of imines 2 and 11-13 were performed following the general procedure for the cyclization of aromatic imines. The crude material was chromatographed on $\mathrm{SiO}_{2}(2-$ $50 \%$ EtOAc-hexanes) to afford compound 6 as a white solid with yields and ee as described in the text. The ee was determined by HPLC analysis; mp 1 19-120 ${ }^{\circ} \mathrm{C}$. ' $\mathrm{H}$ NMR (400 MHz, $\left.\mathrm{CDCl}_{3}\right): \delta=$ $9.82(\mathrm{~s}, 1 \mathrm{H}, \mathrm{CHO}), 7.39(\mathrm{~d}, 1 \mathrm{H}, J=8.3 \mathrm{~Hz}, \mathrm{ArH}), 6.98(\mathrm{~d}, 1 \mathrm{H}$, $J=8.3 \mathrm{~Hz}, \mathrm{ArH}), 6.96-6.62(\mathrm{~m}, 2 \mathrm{H}, \mathrm{ArH} \times 2), 6.82(\mathrm{~d}, 1 \mathrm{H}$, $J=8.2, \operatorname{ArH}), 6.00(\mathrm{~d}, 1 \mathrm{H}, J=10.2 \mathrm{~Hz}, \operatorname{ArOCHAr}), 4.89(\mathrm{~d}, 1 \mathrm{H}$, $\left.J=10.2 \mathrm{~Hz}, \mathrm{ArCHCO}_{2} \mathrm{CH}_{3}\right), 3.97\left(\mathrm{~s}, 3 \mathrm{H}, \mathrm{OCH}_{3}\right), 3.85(\mathrm{~s}, 3 \mathrm{H}$, $\left.\mathrm{OCH}_{3}\right), 3.84\left(\mathrm{~s}, 3 \mathrm{H}, \mathrm{OCH}_{3}\right), 3.23\left(\mathrm{~s}, 3 \mathrm{H}, \mathrm{CO}_{2} \mathrm{CH}_{3}\right) \cdot{ }^{13} \mathrm{C} \mathrm{NMR}(100$ $\left.\mathrm{MHz}, \mathrm{CDCl}_{3}\right): \delta=190.6,169.7,149.9,149.5,149.0,148.5,128.7$,
$128.2,126.4,125.3,119.4,111.5,110.3,109.4,87.7,56.0,55.8$, $55.7,54.1,51.7 .[a]_{\mathrm{D}}{ }^{25}-112.9\left(\mathrm{c} 0.95, \mathrm{CH}_{2} \mathrm{Cl}_{2}\right)$. HRMS-FAB: $\mathrm{m} / \mathrm{z}$ calcd for $\mathrm{C}_{20} \mathrm{H}_{20} \mathrm{O}_{7}[\mathrm{M}]^{+}: 372.1209$; found: 372.1213 . HPLC analysis [AS column, hexanes-i- $\mathrm{PrOH}(70: 30), 40$ min run time]: peak 1 , $t_{\mathrm{R}}=16.6 \mathrm{~min}$ (minor enantiomer) and peak $2, t_{\mathrm{R}}=31.0 \mathrm{~min}$ (major enantiomer).

3-(3-Formylphenoxy)-3-phenylacrylic Acid Methyl Ester

To a sealed tube equipped with a magnetic stir bar was added $\mathrm{MeOH}(42 \mathrm{~mL})$ followed by $\mathrm{Na}$ metal $(1.58 \mathrm{~g}, 68.8 \mathrm{mmol}, 1.6$ equiv, cut into small pieces). Once all the $\mathrm{Na}$ had dissolved, isovanillin ( $48.4 \mathrm{~g}, 318 \mathrm{mmol}, 7.6$ equiv) was added, and the reaction mixture became a thick, yellow slurry. After stirring at r.t. for $5 \mathrm{~min}$, pyridine $(42 \mathrm{~mL})$ was added, followed by phenylpropynoic acid methyl ester $(5.00 \mathrm{~g}, 43.0 \mathrm{mmol}, 1.0$ equiv), and the reaction mixture was stirred at $120^{\circ} \mathrm{C}$ for $1.75 \mathrm{~h}$. The mixture was cooled to r.t., diluted with EtOAc, and sat. aq $\mathrm{NH}_{4} \mathrm{Cl}$ solution was added. The layers were separated, and the organic layer was washed several times with sat. aq $\mathrm{K}_{2} \mathrm{CO}_{3}$ solution to remove excess isovanillin. The combined organic layers were dried, filtered, and concentrated under reduced pressure. The crude material was chromatographed on basic alumina (0-20\% EtOAc-hexane) to afford the $Z$-alkene as a white solid $(5.20 \mathrm{~g}, 18.4 \mathrm{mmol}, 42 \%)$; mp $98-99^{\circ} \mathrm{C} .{ }^{1} \mathrm{H}$ NMR $(400 \mathrm{MHz}$, $\left.\mathrm{CDCl}_{3}\right): \delta=9.68(\mathrm{~s}, 1 \mathrm{H}, \mathrm{CHO}), 7.57(\mathrm{dd}, 2 \mathrm{H}, J=2.0,6.4 \mathrm{~Hz}$, ArH), 7.45 (dd, $1 \mathrm{H}, J=2.0,8.4 \mathrm{~Hz}, \mathrm{ArH}), 7.35-7.25(\mathrm{~m}, 4 \mathrm{H}$, $J=2.0 \mathrm{~Hz}, \mathrm{ArH}), 7.00(\mathrm{~d}, 1 \mathrm{H}, J=8.4 \mathrm{~Hz}, \operatorname{ArH}), 6.15(\mathrm{~s}, 1 \mathrm{H}$, $\mathrm{CHCO}_{2} \mathrm{Me}$ ), $4.00\left(\mathrm{~s}, 3 \mathrm{H}, \mathrm{OCH}_{3}\right), 3.64\left(\mathrm{~s}, 3 \mathrm{H}, \mathrm{OCH}_{3}\right) \cdot{ }^{13} \mathrm{C} \mathrm{NMR}$ $\left(100 \mathrm{MHz}, \mathrm{CDCl}_{3}\right): \delta=190.2,164.3,162.1,154.4,146.5,133.1$, $130.8,129.7,128.7,127.5,126.8,114.9,111.5,105.8,56.2,51.2$. HRMS-FAB: $m / z$ calcd for $\mathrm{C}_{18} \mathrm{H}_{17} \mathrm{O}_{5}[\mathrm{M}+\mathrm{H}]^{+}: 313.1076$; found: 313.1076 .

3-[5-(Indan-1-yliminomethyl)-2-methoxyphenoxy]-3-phenylacrylic Acid Methyl Esters (14)

This compound was prepared according to the general procedure for imine synthesis with 1 equiv of aldehyde ( $14.1 \mathrm{mg}, 0.0500 \mathrm{mmol}$ ) and 1.5 equiv of amine $(7.3 \mathrm{mg}, 0.0550 \mathrm{mmol}){ }^{1} \mathrm{H}$ NMR ( $400 \mathrm{MHz}$, $\left.\mathrm{CDCl}_{3}\right): \delta=8.20(\mathrm{~s}, 1 \mathrm{H}, \mathrm{CH}=\mathrm{NCH}), 7.59(\mathrm{~d}, 2 \mathrm{H}, J=7.6 \mathrm{~Hz}$, ArH $\times 2), 7.43(\mathrm{~d}, 1 \mathrm{H}, J=8.0 \mathrm{~Hz}, \mathrm{ArH}), 7.37-7.32(\mathrm{~m}, 3 \mathrm{H}$, $\operatorname{ArH} \times 3), 7.20-7.16(\mathrm{~m}, 4 \mathrm{H}, \operatorname{ArH} \times 4), 7.02(\mathrm{~d}, 1 \mathrm{H}, J=7.6 \mathrm{~Hz}$, ArH), 6.93 (d, $1 \mathrm{H}, J=8.4 \mathrm{~Hz}, \mathrm{ArH}), 6.05\left(\mathrm{~s}, 1 \mathrm{H}, \mathrm{CHCO}_{2} \mathrm{CH}_{3}\right.$ ), $4.81\left(\mathrm{t}, 1 \mathrm{H}, J=7.2 \mathrm{~Hz}, \mathrm{CH}_{2} \mathrm{CHN}=\mathrm{CH}\right), 3.98\left(\mathrm{~s}, 3 \mathrm{H}, \mathrm{OCH}_{3}\right), 3.68$ (s, $3 \mathrm{H}, \mathrm{OCH}_{3}$ ), 3.12-3.07 (m, l H, one of $\mathrm{ArCH}_{2} \mathrm{CH}_{2}$ ), 2.94-2.88 (m, $1 \mathrm{H}$, one of $\left.\mathrm{ArCH}_{2} \mathrm{CH}_{2}\right), 2.45-2.39\left(\mathrm{~m}, 1 \mathrm{H}\right.$, one of $\mathrm{ArCH}_{2} \mathrm{CH}_{2}$ ), 2.20-2.15 (m, $1 \mathrm{H}$, one of $\left.\mathrm{ArCH}_{2} \mathrm{CH}_{2}\right) .{ }^{13} \mathrm{C}$ NMR $(100 \mathrm{MHz}$, $\left.\mathrm{CDCl}_{3}\right): \delta=164.9,163.0,159.1,151.4,148.1,146.0,133.9,130.5$, $129.7,129.4,128.6,127.1,123.9,120.4,120.4,116.6,113.2,113.1$, $112.0,105.1,71.6,56.2,51.3,34.1,31.4 .[\alpha]_{D}^{25}+1.3$ (c 2.9, $\mathrm{CH}_{2} \mathrm{Cl}_{2}$ ).

3-\{5-[(7-Fluoroindan-1-ylimino)methyl]-2-methoxyphenoxy $\}$ 3-phenylacrylic Acid Methyi Ester (15)

This compound was prepared according to the general procedure for imine synthesis with 1 equiv of aldehyde $(14.1 \mathrm{mg}, 0.0500 \mathrm{mmol})$ and 1.5 equiv of amine $(8.3 \mathrm{mg}, 0.0550 \mathrm{mmol}){ }^{1} \mathrm{H}$ NMR $(400 \mathrm{MHz}$, $\left.\mathrm{CDCl}_{3}\right): \delta=8.12(\mathrm{~s}, 1 \mathrm{H}, \mathrm{CH}=\mathrm{NCH}), 7.59(\mathrm{~d}, 2 \mathrm{H}, J=6.8 \mathrm{~Hz}$, ArH $\times 2), 7.36-7.27(\mathrm{~m}, 4 \mathrm{H}, \mathrm{ArH} \times 4), 7.18-7.13(\mathrm{~m}, 2 \mathrm{H}$, ArH $\times 2), 7.02(\mathrm{~d}, 1 \mathrm{H}, J=5.6 \mathrm{~Hz}, \operatorname{ArH}), 6.87(\mathrm{~d}, 1 \mathrm{H}, J=6.8 \mathrm{~Hz}$, $\mathrm{ArH}), 6.78(\mathrm{t}, 1 \mathrm{H}, J=6.8 \mathrm{~Hz}, \mathrm{ArH}), 6.00\left(\mathrm{~s}, 1 \mathrm{H}, \mathrm{CHCO} \mathrm{CH}_{3}\right), 5.03$ $\left(\mathrm{m}, 1 \mathrm{H}, \mathrm{CH}_{2} \mathrm{CHN}=\mathrm{CH}\right), 3.92\left(\mathrm{~s}, 3 \mathrm{H}, \mathrm{OCH}_{3}\right), 3.65\left(\mathrm{~s}, 3 \mathrm{H}, \mathrm{OCH}_{3}\right)$, 3.20-3.14 (m, $1 \mathrm{H}$, one of $\left.\mathrm{ArCH}_{2} \mathrm{CH}_{2}\right), 2.89-2.85$ (m, I H, one of $\left.\mathrm{ArCH}_{2} \mathrm{CH}_{2}\right), 2.40-2.36\left(\mathrm{~m}, 1 \mathrm{H}\right.$, one of $\left.\mathrm{ArCH}_{2} \mathrm{CH}_{2}\right), 2.17-2.14(\mathrm{~m}$, $1 \mathrm{H}$, one of $\left.\mathrm{ArCH}_{2} \mathrm{CH}_{2}\right) \cdot{ }^{13} \mathrm{C} \mathrm{NMR}\left(100 \mathrm{MHz}, \mathrm{CDCl}_{3}\right): \delta=164.8$ $162.9,159.4,151.5,146.1,144.4,143.7,130.6,129.4,128.6,127.4$, $127.1,126.3,124.7,124.3,123.9,116.4,111.9,105.3,74.7,56.2$, $51.3,34.2,30.9$ (multiple C's coupled to $\mathrm{F}$ ). $[\mathrm{a}]_{\mathrm{D}}{ }^{25}+1.8(\mathrm{c} 1.1$, $\mathrm{CH}_{2} \mathrm{Cl}_{2}$ ). 
7-Formyl-4-methoxy-2-phenylindan-1-carboxylic Acid Methyl Ester (16)

The $\mathrm{C}-\mathrm{H}$ activation reactions of imines 14 and 15 were performed following the general procedure for the cyclization of aromatic imines. The crude material was chromatographed on $\mathrm{SiO}_{2}(2-30 \%$ EtOAc-hexanes) to afford compound 16 as a white solid with yields and ee as described in the text. The ee was determined by HPLC analysis; $\mathrm{mp} 135-136^{\circ} \mathrm{C}$. ' $\mathrm{H} \mathrm{NMR}\left(400 \mathrm{MHz}, \mathrm{CDCl}_{3}\right): \delta=9.82$ (s, $1 \mathrm{H}, \mathrm{CHO}), 7.41-7.28(\mathrm{~m}, 6 \mathrm{H}, \mathrm{ArH} \times 6), 6.99(\mathrm{~d}, 1 \mathrm{H}, J=8.4 \mathrm{~Hz}$, ArH), 6.07 (d, l H, $J=10.4 \mathrm{~Hz}$, ArOCHAr), 4.95 (d, $1 \mathrm{H}, J=10.0$ $\left.\mathrm{Hz}, \mathrm{ArCHCO}_{2} \mathrm{CH}_{3}\right), 3.97\left(\mathrm{~s}, 3 \mathrm{H}, \mathrm{OCH}_{3}\right), 3.13\left(\mathrm{~s}, 3 \mathrm{H}, \mathrm{OCH}_{3}\right) .{ }^{13} \mathrm{C}$ NMR (100 MHz, $\left.\mathrm{CDCl}_{3}\right): \delta=190.7,169.5,150.4,149.6,136.0$, $128.8,128.51,128.1,126.6,126.5,125.3,111.7,87.8,56.2,54.3$, 51.6. HRMS-FAB: $m / z$ calcd for $\mathrm{C}_{18} \mathrm{H}_{16} \mathrm{O}_{5}[\mathrm{M}]^{+}: 312.0998$; found: 312.0998. HPLC analysis [AS column, hexane- $i-\mathrm{PrOH}$ (75:25), 45 min run time]: peak $1, t_{\mathrm{R}}=10.8 \mathrm{~min}$ (minor enantiomer) and peak $2, t_{R}=20.0$ min (major enantiomer).

\section{3-Isopropenyloxybenzaldehyde}

To a $100 \mathrm{~mL}$ round-bottom flask equipped with a magnetic stir bar and septum were added 1.5 equiv $(7.98 \mathrm{~g}, 24.6 \mathrm{mmol})$ of $\mathrm{Cs}_{2} \mathrm{CO}_{3}$, 0.25 equiv of $\mathrm{CuCl}(405 \mathrm{mg}, 4.10 \mathrm{mmol}$ ), 0.5 equiv of acetylacetone ( $819 \mathrm{mg}, 8.19 \mathrm{mmol}$ ), and $50 \mathrm{~mL}$ of THF. The suspension was stirred for 5 min at r.t. Under an argon atmosphere, 3-hydroxybenzaldehyde $(2.0 \mathrm{~g}, 16.38 \mathrm{mmol})$ and 1.3 equiv of 2-bromopropene $(1.86 \mathrm{~mL}, 21.29 \mathrm{mmol})$ were added to the reaction mixture. The reaction flask was then equipped with a reflux condenser, and the mixture was heated to reflux for $12 \mathrm{~h}$. The reaction mixture was cooled to r.t., filtered, and concentrated under reduced pressure. The crude material was chromatographed on $\mathrm{SiO}_{2}(0-10 \%$ EtOAc-hexanes) to afford the title compound as a yellow oil $(690 \mathrm{mg}, 4.26$ mmol, $26 \%$ ). ${ }^{1} \mathrm{H} \mathrm{NMR}\left(400 \mathrm{MHz}, \mathrm{CDCl}_{3}\right.$ ): $\delta=9.92$ (s, $1 \mathrm{H}, \mathrm{CHO}$ ), 7.56 (dd, $1 \mathrm{H}, J=1.2,10.0 \mathrm{~Hz}, \mathrm{ArH}), 7.49(\mathrm{t}, 1 \mathrm{H}, J=1.6 \mathrm{~Hz}, \mathrm{ArH}$ ), 7.44 (t, $1 \mathrm{H}, J=7.6 \mathrm{~Hz}, \mathrm{ArH}), 7.24$ (dd, $1 \mathrm{H}, J=1.2,10.8 \mathrm{~Hz}, \mathrm{ArH}$ ), 4.23 (d, $\left.1 \mathrm{H}, J=2.0 \mathrm{~Hz}, \mathrm{CH}_{2}=\mathrm{CCH}_{3}\right), 3.99(\mathrm{~d}, 1 \mathrm{H}, J=2.0 \mathrm{~Hz}$, $\left.\mathrm{CH}_{2}=\mathrm{CCH}_{3}\right), 1.93\left(\mathrm{~s}, 3 \mathrm{H}, \mathrm{CH}_{2}=\mathrm{CCH}_{3}\right) .{ }^{13} \mathrm{C}$ NMR $(100 \mathrm{MHz}$, $\left.\mathrm{CDCl}_{3}\right): \delta=191.3,158.7,156.0,137.8,130.0,126.4,125.2,120.1$, 91.5, 19.5. HRMS-FAB: $m / z$ caled for $\mathrm{C}_{10} \mathrm{H}_{10} \mathrm{O}_{2}[\mathrm{M}]^{+}: 162.0681$; found: 162.0681 .

\section{3-Propenyloxybenzaldehyde}

To a $100 \mathrm{~mL}$ round-bottom flask equipped with a magnetic stir bar and septum were added 1.5 equiv (7.98 g, $24.6 \mathrm{mmol}$ ) of $\mathrm{Cs}_{2} \mathrm{CO}_{3}$, 0.25 equiv of $\mathrm{CuCl}(405 \mathrm{mg}, 4.10 \mathrm{mmol}), 0.5$ equiv of acetylacetone $(819 \mathrm{mg}, 8.19 \mathrm{mmol}$ ), and $50 \mathrm{~mL}$ of THF. The suspension was stirred for $5 \mathrm{~min}$ at r.t. Under an argon atmosphere, 1 equiv of 3[1,3]dioxolan-2-yl-phenol $(2.72 \mathrm{~g}, 16.38 \mathrm{mmol})$ and 1.5 equiv of $1-$ bromopropene were added to the reaction mixture. The reaction flask was then equipped with a reflux condenser and the mixture was heated to reflux for $12 \mathrm{~h}$. The reaction mixture was cooled to r.t., filtered, and concentrated under reduced pressure. The acetal was hydrolyzed by stirring in $6 \mathrm{~N} \mathrm{HCl-MeOH}$ for $1 \mathrm{~h}$. The mixture was extracted with EtOAc and concentrated, and the crude material was chromatographed on $\mathrm{SiO}_{2}(0-10 \%$ EtOAc-hexanes) to afford the $E$-alkene as a yellow oil ( $584 \mathrm{mg}, 3.60 \mathrm{mmol}, 22 \%$ ). 'H NMR $\left(400 \mathrm{MHz}, \mathrm{CDCl}_{3}\right): \delta=9.90(\mathrm{~s}, 1 \mathrm{H}, \mathrm{CHO}), 7.45$ (dd, $1 \mathrm{H}, J=1.2$, $8.0 \mathrm{~Hz}, \mathrm{ArH}), 7.41-7.37(\mathrm{~m}, 2 \mathrm{H}, \mathrm{ArH}), 7.16(\mathrm{dd}, 1 \mathrm{H}, J=1.2,5.2$ $\mathrm{Hz}, \mathrm{ArH}), 6.40-6.35\left(\mathrm{~m}, 1 \mathrm{H}, \mathrm{CH}_{3} \mathrm{CH}=\mathrm{CH}\right), 5.42-5.34(\mathrm{~m}, 1 \mathrm{H}$, $\left.\mathrm{CH}_{3} \mathrm{CH}=\mathrm{CH}\right), 1.63\left(\mathrm{dd}, 3 \mathrm{H}, J=1.6,6.8 \mathrm{~Hz}, \mathrm{CH}_{3} \mathrm{CH}=\mathrm{CH}\right) .{ }^{13} \mathrm{C}$ NMR $\left(100 \mathrm{MHz}, \mathrm{CDCl}_{3}\right): \delta=191.6,157.8,140.9,137.7,130.1$, $124.4,122.6,114.9,109.9,12.1$. HRMS-FAB $(+): \mathrm{m} / \mathrm{z}$ calcd for $\mathrm{C}_{10} \mathrm{H}_{10} \mathrm{O}_{2}[\mathrm{M}]^{+}: 162.0681$; found: 162.0680 .

Imines 17-20 were prepared according to the general procedure for imine synthesis.
Indan-1-yl-(3-isopropenyloxybenzylidene)amine (17)

One equiv of aldehyde $(8.1 \mathrm{mg}, 0.0500 \mathrm{mmol})$ and 1.5 equiv of amine $(7.32 \mathrm{mg}, 0.0550 \mathrm{mmol}$ ) were condensed. 'H NMR (400 $\left.\mathrm{MHz}, \mathrm{CDCl}_{3}\right): \delta=8.43(\mathrm{~s}, 1 \mathrm{H}, \mathrm{CH}=\mathrm{NCH}), 7.55(\mathrm{~d}, 1 \mathrm{H}, J=7.6 \mathrm{~Hz}$, ArH), 7.51 (s, $1 \mathrm{H}, \mathrm{ArH}), 7.39$ (t, $1 \mathrm{H}, J=8.0 \mathrm{~Hz}, \mathrm{ArH}), 7.30$ (d, 1 $\mathrm{H}, J=7.2 \mathrm{~Hz}, \mathrm{ArH}), 7.26-7.13(\mathrm{~m}, 2 \mathrm{H}, \mathrm{ArH} \times 2), 7.12(\mathrm{~m}, 2 \mathrm{H}$, $\mathrm{ArH} \times 2), 4.95\left(\mathrm{t}, 1 \mathrm{H}, J=7.2 \mathrm{~Hz}, \mathrm{CH}_{2} \mathrm{CHN}=\mathrm{CH}\right), 4.21[\mathrm{~s}, 1 \mathrm{H}$, one of $\left.\mathrm{CH}_{2}=\mathrm{C}(\mathrm{O}) \mathrm{CH}_{3}\right], 4.01\left[\mathrm{~s}, 1 \mathrm{H}\right.$, one of $\left.\mathrm{CH}_{2}=\mathrm{C}(\mathrm{O}) \mathrm{CH}_{3}\right], 3.18-3.12$ (m, $1 \mathrm{H}$, one of $\left.\mathrm{ArCH}_{2} \mathrm{CH}_{2}\right), 3.02-2.94\left(\mathrm{~m}, 1 \mathrm{H}\right.$, one of $\mathrm{ArCH}_{2} \mathrm{CH}_{2}$ ), 2.53-2.47 (m, $1 \mathrm{H}$, one of $\left.\mathrm{ArCH}_{2} \mathrm{CH}_{2}\right), 2.32-2.23(\mathrm{~m}, 1 \mathrm{H}$, one of $\left.\mathrm{ArCH}_{2} \mathrm{CH}_{2}\right), 2.00\left(\mathrm{~s}, 3 \mathrm{H}, \mathrm{CH}_{3}\right) .{ }^{13} \mathrm{C} \mathrm{NMR}\left(100 \mathrm{MHz}, \mathrm{CDCl}_{3}\right): \delta=$ $159.8,159.4,155.7,144.1,143.8,138.0,129.6,127.6,126.4,124.8$, $124.3,124.0,123.0,120.1,90.3,74.9,34.2,31.0,20.0 .[\alpha]_{D}{ }^{25}+14.6$ (c $1.0, \mathrm{CH}_{2} \mathrm{Cl}_{2}$ ).

(7-Fluoroindan-1-yl)-(3-isopropenyloxybenzylidene)amine (18) One equiv of aldehyde $(8.3 \mathrm{mg}, 0.0500 \mathrm{mmol})$ and 1.5 equiv of amine ( $7.3 \mathrm{mg}, 0.0550 \mathrm{mmol}$ ) were condensed. 'H NMR ( $400 \mathrm{MHz}$, $\left.\mathrm{CDCl}_{3}\right): \delta=8.48(\mathrm{~s}, 1 \mathrm{H}, \mathrm{CH}=\mathrm{NCH}), 7.50-7.43(\mathrm{~m}, 2 \mathrm{H}, \mathrm{ArH} \times 2)$, $7.34(\mathrm{t}, 1 \mathrm{H}, J=10.4 \mathrm{~Hz}, \mathrm{ArH}), 7.25-7.17$ (m, $1 \mathrm{H}, \mathrm{ArH}), 7.09-7.05$ $(\mathrm{m}, 2 \mathrm{H}, \mathrm{ArH} \times 2), 6.82(\mathrm{t}, 1 \mathrm{H}, J=11.2 \mathrm{~Hz}, \mathrm{ArH}), 5.17-5.15(\mathrm{~m}, 1$ $\left.\mathrm{H}, \mathrm{CH}_{2} \mathrm{CHN}=\mathrm{CH}\right), 4.17\left[\mathrm{~s}, 1 \mathrm{H}\right.$, one of $\left.\mathrm{CH}_{2}=\mathrm{C}(\mathrm{O}) \mathrm{CH}_{3}\right], 3.97[\mathrm{~s}, 1$ $\mathrm{H}$, one of $\left.\mathrm{CH}_{2}=\mathrm{C}(\mathrm{O}) \mathrm{CH}_{3}\right], 3.30-3.20\left(\mathrm{~m}, 1 \mathrm{H}\right.$, one of $\mathrm{ArCH} \mathrm{CH}_{2}$ ), 2.99-2.89 (m, $1 \mathrm{H}$, one of $\left.\mathrm{ArCH}_{2} \mathrm{CH}_{2}\right), 2.51-2.41(\mathrm{~m}, 1 \mathrm{H}$, one of $\left.\mathrm{ArCH}_{2} \mathrm{CH}_{2}\right), 2.29-2.23\left(\mathrm{~m}, 1 \mathrm{H}\right.$, one of $\left.\mathrm{ArCH}_{2} \mathrm{CH}_{2}\right), 1.97(\mathrm{~s}, 3 \mathrm{H}$, $\left.\mathrm{CH}_{3}\right) \cdot[\alpha]_{\mathrm{D}}{ }^{25}+2.2\left(\mathrm{c} 1.3, \mathrm{CH}_{2} \mathrm{Cl}_{2}\right)$.

\section{Indan-1-yl-(3-propenyloxybenzylidene)amine (19)}

One equiv of aldehyde $(8.1 \mathrm{mg}, 0.0500 \mathrm{mmol})$ and 1.5 equiv of amine (7.3 $\mathrm{mg}, 0.0550 \mathrm{mmol})$ were condensed. 'H NMR (400 MHz, $\mathrm{CDCl}_{3}$ ): $\delta=8.43$ (s, $\left.1 \mathrm{H}, \mathrm{CH}=\mathrm{NCH}\right), 7.49$ (s, $\left.1 \mathrm{H}, \mathrm{ArH}\right), 7.43$ (d, 1 $\mathrm{H}, J=6.0 \mathrm{~Hz}, \mathrm{ArH}), 7.36(\mathrm{t}, 1 \mathrm{H}, J=6.0 \mathrm{~Hz}, \mathrm{ArH}), 7.32(\mathrm{~d}, 1 \mathrm{H}$, $J=5.6 \mathrm{~Hz}, \mathrm{ArH}), 7.26(\mathrm{t}, 1 \mathrm{H}, J=5.2 \mathrm{~Hz}, \mathrm{ArH}), 7.21(\mathrm{t}, 1 \mathrm{H}, J=6.0$ $\mathrm{Hz}, \mathrm{ArH}), 7.12(\mathrm{~d}, 1 \mathrm{H}, J=5.6 \mathrm{~Hz}, \mathrm{ArH}), 7.07(\mathrm{~d}, 1 \mathrm{H}, J=6.0 \mathrm{~Hz}$, ArH), 6.49 [d, I H, $\left.J=9.6 \mathrm{~Hz}, \mathrm{HC}\left(\mathrm{CH}_{3}\right)=\mathrm{CHOAr}\right], 5.45-5.38[\mathrm{~m}$, $\left.1 \mathrm{H}, \mathrm{HC}\left(\mathrm{CH}_{3}\right)=\mathrm{CHOAr}\right], 4.96\left(\mathrm{t}, 1 \mathrm{H}, J=5.6 \mathrm{~Hz}, \mathrm{CH}_{2} \mathrm{CHN}=\mathrm{CH}\right)$, $3.18-3.13\left(\mathrm{~m}, 1 \mathrm{H}\right.$, one of $\left.\mathrm{ArCH}_{2} \mathrm{CH}_{2}\right), 3.02-2.96(\mathrm{~m}, 1 \mathrm{H}$, one of $\left.\mathrm{ArCH}_{2} \mathrm{CH}_{2}\right), 2.51-2.49\left(\mathrm{~m}, 1 \mathrm{H}\right.$, one of $\left.\mathrm{ArCH}_{2} \mathrm{CH}_{2}\right), 2.33-2.26(\mathrm{~m}$, $1 \mathrm{H}$, one of $\left.\mathrm{ArCH}_{2} \mathrm{CH}_{2}\right), 1.69$ (d, $3 \mathrm{H}, J=5.6 \mathrm{~Hz}, \mathrm{CH}_{3}$ ). ${ }^{13} \mathrm{C} \mathrm{NMR}$ $\left(100 \mathrm{MHz}, \mathrm{CDCl}_{3}\right): \delta=160.0,157.7,144.1,143.8,141.7,137.8$, $129.7,127.6,126.4,124.8,124.3,123.0,119.1,114.6,108.7,74.9$, $34.2,31.0,12.2 .[\alpha]_{D}{ }^{25}+2.1\left(c 1.9, \mathrm{CH}_{2} \mathrm{Cl}_{2}\right)$.

(7-Fluoroindan-1-yl)-(3-propenyloxybenzylidene)amine (20) One equiv of aldehyde $(8.11 \mathrm{mg}, 0.05 \mathrm{mmol})$ and 1.5 equiv of amine (8.31 mg, $0.055 \mathrm{mmol}$ ) were condensed. 'H NMR (400 MHz, $\left.\mathrm{CDCl}_{3}\right): \delta=8.39(\mathrm{~s}, 1 \mathrm{H}, \mathrm{CH}=\mathrm{NCH}), 7.40-7.37(\mathrm{~m}, 2 \mathrm{H}, \mathrm{ArH} \times 2)$, 7.32 (t, $1 \mathrm{H}, J=7.6 \mathrm{~Hz}, \mathrm{ArH}), 7.25-7.16(\mathrm{~m}, 1 \mathrm{H}, \mathrm{ArH}), 7.08$ (d, $\mathrm{H}, J=7.2 \mathrm{~Hz}, \mathrm{ArH}), 7.02(\mathrm{~d}, 1 \mathrm{H}, J=8.0 \mathrm{~Hz}, \mathrm{ArH}), 6.84(\mathrm{t}, 1 \mathrm{H}$, $J=8.8 \mathrm{~Hz}, \mathrm{ArH}), 6.46\left[\mathrm{~d}, 1 \mathrm{H}, J=12.4 \mathrm{~Hz}, \mathrm{HC}\left(\mathrm{CH}_{3}\right)=\mathrm{CHOAr}\right]$, 5.41-5.36 [m, 1 H, HC(CH $)=\mathrm{CHOAr}], 5.18-5.17(\mathrm{~m}, 1 \mathrm{H}$, $\left.\mathrm{CH}_{2} \mathrm{CHN}=\mathrm{CH}\right), 3.31-3.23\left(\mathrm{~m}, 1 \mathrm{H}\right.$, one of $\left.\mathrm{ArCH}_{2} \mathrm{CH}_{2}\right), 2.98-2.97$ (m, $1 \mathrm{H}$, one of $\left.\mathrm{ArCH}_{2} \mathrm{CH}_{2}\right), 2.52-2.47$ (m, $1 \mathrm{H}$, one of $\mathrm{ArCH}_{2} \mathrm{CH}_{2}$ ), 2.29-2.28 (m, $1 \mathrm{H}$, one of $\left.\mathrm{ArCH}_{2} \mathrm{CH}_{2}\right), 1.68[\mathrm{~d}, 3 \mathrm{H}, J=6.8 \mathrm{~Hz}$, $\left.\left.\mathrm{C}\left(\mathrm{CH}_{3}\right)_{3}\right] .{ }^{13} \mathrm{C} \mathrm{NMR}(100 \mathrm{MHz}, \mathrm{CDCl})_{3}\right): \delta=159.9,159.2,157.7$, $148.2,148.1,141.7,137.9,130.0,129.9,129.9,129.6,122.9,120.5$, $120.5,119.0,114.8,113.3,113.1,108.6,71.8,34.1,31.5,31.5,12.3$ (multiple C's coupled to $F$ ). $[\alpha]_{\mathrm{D}}{ }^{25}+6.0\left(\mathrm{c} 1.0, \mathrm{CH}_{2} \mathrm{Cl}_{2}\right)$.

The C-H activation reactions of imines 17-20 were prepared following the general procedure for the cyclization of aromatic imines. The crude material was chromatographed on $\mathrm{SiO}_{2}(0-1 \%$ EtOAchexanes) to afford compounds 21 and 22 as colorless oils with yields and ee as described in the text. The ee was determined by HPLC analysis. 
2-Methyl-2,3-dihydrobenzofuran-4-carbaldehyde (21)

' $\mathrm{H}$ NMR (400 MHz, $\mathrm{CDCl}_{3}$ ): $\delta=10.03$ (s, $1 \mathrm{H}, \mathrm{CHO}$ ), 7.30-7.26 ( $\mathrm{m}, 2 \mathrm{H}, \mathrm{ArH} \times 2), 7.01-6.98(\mathrm{~m}, 1 \mathrm{H}, \mathrm{ArH}), 5.06-4.97[\mathrm{~m}, 1 \mathrm{H}$, $\mathrm{CH}_{3} \mathrm{CH}\left(\mathrm{CH}_{2}\right) \mathrm{OAr}$ ], $3.70-3.64$ [dd, $1 \mathrm{H}, J=17.6,8.8 \mathrm{~Hz}$, one of $\mathrm{CH}_{3} \mathrm{CH}\left(\mathrm{CH}_{2}\right) \mathrm{OAr}$ ], 3.15-3.09 [dd, $1 \mathrm{H}, J=17.6,7.6 \mathrm{~Hz}$, one of $\left.\mathrm{CH}_{3} \mathrm{CH}\left(\mathrm{CH}_{2}\right) \mathrm{OAr}\right], 1.48$ (d, $3 \mathrm{H}, J=6.0 \mathrm{~Hz}, \mathrm{CH}_{3} \mathrm{CH}$ ) ${ }^{13} \mathrm{C}$ NMR $\left(100 \mathrm{MHz}, \mathrm{CDCl}_{3}\right): \delta=192.5,160.6,132.9,128.5,128.0,124.1$, $114.7,80.7,36.8,21.8$. HPLC analysis [AS column, hexanes$i-\operatorname{PrOH}(99: 1), 20$ min run time]: peak $1, t_{\mathrm{R}}=9.3 \mathrm{~min}$ (minor enantiomer) and peak $2, t_{\mathrm{R}}=8.9$ min (major enantiomer).

3-Methyl-2,3-dihydrobenzofuran-4-carbaldehyde (22)

${ }^{\prime} \mathrm{H}$ NMR (400 MHz, $\mathrm{CDCl}_{3}$ ): $\delta=10.06$ (s, $1 \mathrm{H}, \mathrm{CHO}$ ), 7.34-7.28 $(\mathrm{m}, 2 \mathrm{H}, \mathrm{ArH} \times 2), 7.05-7.02(\mathrm{~m}, 1 \mathrm{H}, \mathrm{ArH}), 4.60[\mathrm{t}, 1 \mathrm{H}, J=11.2$ $\left.\mathrm{Hz}, \quad \mathrm{CH}_{3} \mathrm{CH}\left(\mathrm{CH}_{2}\right) \mathrm{OAr}\right], \quad 4.34-4.29 \quad[\mathrm{~m}, \quad \mathrm{H} \mathrm{H}$, one of $\left.\mathrm{CH}_{3} \mathrm{CH}\left(\mathrm{CH}_{2}\right) \mathrm{OAr}\right], 3.98-3.92\left[\mathrm{~m}, 1 \mathrm{H}\right.$, one of $\left.\mathrm{CH}_{3} \mathrm{CH}\left(\mathrm{CH}_{2}\right) \mathrm{OAr}\right]$, 1.30 (d, $\left.3 \mathrm{H}, J=9.2 \mathrm{~Hz}, \mathrm{CH}_{3} \mathrm{CH}\right) .{ }^{13} \mathrm{C} \mathrm{NMR}\left(100 \mathrm{MHz}, \mathrm{CDCl}_{3}\right)$ : $\delta=192.1,160.6,133.4,132.7,128.7,124.8,115.2,79.3,36.1,20.5$. HPLC analysis [AS column, hexanes- $i$-PrOH $(99: 1), 20 \mathrm{~min}$ run time]: peak $1, t_{\mathrm{R}}=10.0 \mathrm{~min}$ (minor enantiomer) and peak $2, t_{\mathrm{R}}=8.9$ min (major enantiomer)

tert-Butyl-(3-isopropenyloxybenzylidene)amine (23)

Imine 23 was prepared following the general procedure for imine formation. ' $\mathrm{H}$ NMR (400 $\left.\mathrm{MHz}, \mathrm{CDCl}_{3}\right)$ ) $\delta=8.22(\mathrm{~s}, 1 \mathrm{H}$, $\mathrm{CH}=\mathrm{NCH}), 7.49(\mathrm{~d}, 1 \mathrm{H}, J=7.6 \mathrm{~Hz}, \mathrm{ArH}), 7.44(\mathrm{~s}, 1 \mathrm{H}, \mathrm{ArH})$, $7.34(\mathrm{t}, 1 \mathrm{H}, J=8.0 \mathrm{~Hz}, \mathrm{ArH}), 7.06(\mathrm{~d}, 1 \mathrm{H}, J=7.6 \mathrm{~Hz}, \mathrm{ArH}), 4.17$ [s, $1 \mathrm{H}$, one of $\left.\mathrm{CH}_{2}=\mathrm{C}\left(\mathrm{CH}_{3}\right) \mathrm{OAr}\right], 3.97[\mathrm{~s}, 1 \mathrm{H}$, one of $\left.\mathrm{CH}_{2}=\mathrm{C}\left(\mathrm{CH}_{3}\right) \mathrm{OAr}\right], 1.98\left(\mathrm{~s}, 3 \mathrm{H}, \mathrm{CCH}_{3}\right), 1.28\left[\mathrm{~s}, 9 \mathrm{H}, \mathrm{C}\left(\mathrm{CH}_{3}\right)_{3}\right] .{ }^{13} \mathrm{C}$ NMR $\left(100 \mathrm{MHz}, \mathrm{CDCl}_{3}\right): \delta=159.4,155.6,154.4,138.9,129.5$, $123.5,122.5,119.8,89.9,57.3,29.6,20.0$. HRMS-FAB: $\mathrm{m} / \mathrm{z}$ calcd for $\mathrm{C}_{14} \mathrm{H}_{20} \mathrm{NO}[\mathrm{M}+\mathrm{H}]^{+}: 218.1545$; found: 218.1545 .

\section{Acknowledgment}

This work was supported by the NIH, GM069559 (to J.A.E.) and 5F32GM071207-02 (to S.O.M.), by the German Academic Exchange Service - DAAD (to A.W.), and by the Director and Office of Energy Research, Office of Basic Energy Sciences, Chemical Sciences Division, U.S. Department of Energy, under contract DEAC03-76SF00098 (to R.G.B.). This work was done with support from the U.S. Department of Energy under Contract No. DE-AC02$05 \mathrm{CH} 11231$.

\section{References and Notes}

(1) For recent reviews of $\mathrm{C}-\mathrm{H}$ activation, see: (a) Alberico, D. Scott, M. E.; Lautens, M. Chem. Rev. 2007, 107, 174.

(b) Kakiuchi, F.; Chatani, N. Top. Organomet. Chem. 2004, 11, 45. (c) Labinger, J. A.; Bercaw, J. E. Nature (London) 2002, 417, 507. (d) Ritleng, V.; Sirlin, C.; Pfeffer, M. Chem. Res. 2003, 102, 1731. (e) Jia, C. G.; Kitamura, T.; Fujiwara, Y. Acc. Chem. Res. 2001, 34, 633.

(2) (a) Thalji, R. K.; Ahrendt, K. A.; Bergman, R. G.; Ellman, J. A. J. Am. Chem. Soc. 2001, 123, 9692. (b) Ahrendt, K. A.; Bergman, R. G.; Ellman, J. A. Org. Lett. 2003, 5, 1301.

(3) (a) Thalji, R. K.; Bergman, R. G.; Ellman, J. A. J. Am. Chem. Soc. 2004, 126, 7192. (b) Wilson, R. M.; Thalji, R. K.; Bergman, R. G.; Ellman, J. A. Org. Lett. 2006, 8, 1745.

(4) O'Malley, S. J.; Tan, K. L.; Watzke, A.; Bergman, R. G.; Ellman, J. A. J. Am. Chem. Soc. 2005, 127, 13496.

(5) (a) Kelley, C. J.; Mahajan, J. R.; Brooks, L. C.; Neubert, L. A.; Breneman, W. R.; Carmack, M. J. Org. Chem. 1975, 40, 1804. (b) Kelley, C. J.; Harruff, R. C.; Carmack, M. J. Org. Chem. 1976, 4l, 449.

(6) Tanuwidjaja, J.; Peltier, H. M.; Ellman, J. A. J. Org. Chem. 2007, 72, 626.

(7) Nguyen, P.; Corpuz, E.; Heidelbaugh, T. M.; Chow, K.; Garst, M. E. J. Org. Chem. 2003, 68, 10195.

(8) Yin, J.; Rainka, M. P.; Zhang, X.-X.; Buchwald, S. L. J. Ain. Chem. Soc. 2002, 124, 2892.

(9) $\mathrm{Ti}(\mathrm{OPh})_{4}$ was prepared in situ by the addition of 4 equiv of phenol to 1 equiv of $\mathrm{Ti}(\mathrm{O} i-\mathrm{Pr})_{4}$ in THF at r.t. Ti(OPh $)_{4}$ was obtained as an orange solid after removal of the solvent.

(10) For an example of $\mathrm{C}-\mathrm{H}$ activation with transimination, see: Jun, C. H.; Moon, C. W.; Lee, D. Y. Chem. Eur. J. 2002, 8, 2422.

(11) Van der Ent, A.; Onderdelinden, A. L. Inorg. Synth. 1973, 14,92 .

(12) Guillaneaux, D.; Kagan, H. J. Am. Chem. Soc. 1995, 60, 2502.

(13) Still, W. C.; Kahn, M.; Mitra, A. J. Org. Chem. 1978, 43, 292. 